\title{
Comparison between simultaneous versus staged bilateral total knee arthroplasty: a prospective, randomized, controlled study
}

\author{
Rajesh Kumar Sharma*, R. P. Meena
}

Department of Orthopaedics, Govt. Medical College, Kota, Rajasthan, India

Received: 21 January 2020

Accepted: 17 March 2020

\section{*Correspondence:}

Dr. Rajesh Kumar Sharma,

E-mail: drrajeshsharma1986@gmail.com

Copyright: ( $)$ the author(s), publisher and licensee Medip Academy. This is an open-access article distributed under the terms of the Creative Commons Attribution Non-Commercial License, which permits unrestricted non-commercial use, distribution, and reproduction in any medium, provided the original work is properly cited.

\section{ABSTRACT}

Background: Bilateral total knee arthroplasty (TKA) is a common procedure nowadays. Although, staging of surgery is a subject of debate. We conducted a study to compare safety and functional outcomes of simultaneous and staged bilateral TKA.

Methods: Our study includes total 70 patients of symptomatic severe bilateral osteoarthritis, underwent simultaneous or staged bilateral TKA during 2015 to 2019. 35 patients were randomly allotted for each procedure. The postoperative evaluations were done according to Knee Society Score at one, three, six and 12 months and yearly thereafter for 2 years following a simultaneous bilateral TKA (group A) and the second procedure in the staged bilateral TKA (group B). In the staged group, the patients were followed at monthly intervals until the second procedure. The categorical variables were statistically significant when $p$ value $<0.05$.

Results: As compared to staged procedure (group B), estimated blood loss was significantly less in simultaneous TKA (group A). Although, blood transfusion rate was significantly high in group A. The length of hospital stay was significantly shorter in group A. Overall complication rate (inpatient and/or 90 days readmission) was not significantly higher in group A. Knee infection rate was significantly lower in simultaneous TKA group. There was no revision of surgery and no mortality in any of our study group within 2 years of follow-up.

Conclusions: Simultaneous bilateral TKA is safe and cost-effective procedure with acceptable complication rates for bilateral symptomatic end stage knee osteoarthritis.

Keywords: Bilateral total knee arthroplasty, Simultaneous, Staged, Knee society score

\section{INTRODUCTION}

Osteoarthritis is the most common cause of knee pain and one of the leading causes of disability worldwide. ${ }^{1,2}$ With increasing life expectancy and prevalence of obesity, the incidence of osteoarthritis is likely to keep increasing. 3,4 Total knee arthroplasty (TKA) is the gold standard treatment choice for end stage osteoarthritis of knee. ${ }^{5}$ In severely deformed bilateral knees, unilateral TKA does not fully restore a patient's function, and results in poorer outcome. These patients frequently require bilateral TKA. Based on clinical and patient factors, bilateral TKA can be performed simultaneously under a same anaesthetic and one-time hospitalization or as staged procedures under separate anaesthetics and separate hospital admissions. Although, the staging or optimal timing of surgery in patients requiring bilateral TKA is controversial and continues to be debated. The aim of our study was to compare outcomes of simultaneous versus staged bilateral TKA in severe end stage bilateral symptomatic knee osteoarthritis and to access relative risks and benefits of each procedure.

\section{METHODS}

After obtaining approval of our institutional research ethics committee, a prospective randomized controlled study was conducted during 2015 to 2019 in department of 
Orthopaedics, Government Medical College, Kota (Rajasthan). Written informed consent was taken from all patients involved in the study. A prospective, randomized, controlled study was designed. A sample size, of 70 patients with bilateral end stage knee osteoarthritis met with inclusion criteria, was taken for study. Study population was further divided randomly using sealed envelopes on the day preceding the surgery to have simultaneous (Group A) or staged bilateral TKA (Group B). 35 patients were taken in each group. An interval ranging from three to six months was taken between the two surgeries in Group B (staged bilateral TKA) in order to allow functional recovery and rehabilitation.

\section{Inclusion criteria}

Patients with severe end stage bilateral symptomatic knee osteoarthritis of either sex, patients with low risk of anaesthesia that was ASA score (American Society of Anaesthesiologist) grade 1 and 2 , and willingness to participate in the study.

\section{Exclusion criteria}

Patients with ASA score grade 3 and 4, significant cardiac comorbidities, previous history of thromboembolism, uncontrolled diabetes, malignant hypertension, high grade chronic obstructive pulmonary disease, and previous knee infection, revision TKA, and patients who were unwilling to undergo simultaneous bilateral TKA.

All patients underwent bilateral TKA with same arthroplasty surgeon's team. The standard medial parapatellar approach was applied in all cases. Same arthroplasty model (PCL sacrificing type LCS design of DePuy, Stryker, and Zimmer) was used. Similar preoperative and postoperative protocols were followed in both groups. All the surgeries were performed under combined epidural spinal anaesthesia in a laminar airflow operating room. Same type of intraoperative antibiotics and postoperative venous thromboembolism prophylaxis was administered to all patients of both the groups. All patients were catheterised for urine output calculation. The urinary catheter was removed 24 hours after surgery. In group A, before a bilateral procedure, two tables with draping equipment were set up and placed next to the laminar flow unit. One table was left covered during the first operation for later use on the opposite side. Both knees were prepared at the same time.

Bilateral TKA was done via standard medial parapatellar approach and patella was resurfaced in all patients of both groups. One suction drain was placed in each knee for post-operative drainage. To reduce peri-operative blood loss, tranexamic acid was given intravenously after the incision $(15 \mathrm{mg} / \mathrm{kg})$ and then 6 hourly $(10 \mathrm{mg} / \mathrm{kg})$ for next 12 hours in both groups. Each patient of both groups received $1 \mathrm{~g}$ of Ceftriaxone intravenously 30 minutes prior to the surgical incision followed by $1 \mathrm{~g}$ twice daily postoperatively for one week. Subcutaneous enoxaparin
(40 mg once daily) was given to all patients from the day of surgery to one week postoperatively for the prophylaxis of deep vein thrombosis (DVT). Oral aspirin (150 mg once daily) was given for three to four weeks after the discontinuation of enoxaparin. Doppler ultrasonography was carried out if DVT was suspected. Early mobilisation was done both to prevent DVT and to improve functional recovery. The average surgical time, perioperative blood loss and length of hospital stay in both groups were recorded. Post-operatively, the haematocrit was measured at eight hours and the hemoglobin levels on the first, second and third post-operative days. Blood transfusion requirement was assessed according to intra-operative blood loss, clinical and haematological parameters in both the groups.

Complications local to each joint including superficial wound infection, deep wound infection around the prosthesis, and revision surgery rates within 2 years were recorded. Systemic complications including significant cardiac morbidity, cerebrovascular accidents, confusion, deep vein thrombosis, pulmonary embolism, and urinary tract infection were noted in both groups. The patients had clinical and radiological evaluation at one, three, six and 12 months and yearly thereafter for 2 years following a simultaneous bilateral TKA Group (A) and the second procedure in the staged bilateral TKA Group (B). Those patients in the staged group were followed at monthly intervals until the second procedure. Functional outcome (ability to walk, sit cross-legged position, and climb stairs) was evaluated pre- and post-operatively, using the knee society score (KSS).

\section{Statistical analysis}

Statistical analysis was undertaken using SPSS v10.0 and MS excel 2013 with the independent sample t-test for continuous variables and the chi-squared test for categorical variables. The level of significance applied was $95.0 \%$ ( $\mathrm{p}$ value $<0.05$ ). The measurements were expressed as mean and standard deviation for continuous variables and percentages for categorical variables.

\section{RESULTS}

In our study, all demographic data (age, sex, BMI, comorbidity) were comparable in both groups. The mean surgical time in Group A (simultaneous bilateral TKA) and Group B (staged bilateral TKA) were 208.17 and 217.3 minutes, respectively and this difference was not statistically significant ( $p$ value 0.7485 ). The mean estimated blood loss in group A was $765.5 \mathrm{ml}$ and in Group B was $1090.8 \mathrm{ml}$, and the difference was statistically significant ( $\mathrm{p}$ value $<0.001$ ). The average blood transfusion rate for Group A and Group B were $39.4 \%$ and $18.1 \%$, respectively and the difference was statistically significant ( $p$ value $<0.0001$ ). In this study, the 'in-patient' length of hospital stay was 5.5 mean days for 'simultaneous bilateral TKA group and 8.5 mean days for 'staged bilateral TKA group. The differences in in-patient 
length of hospital stay was statistically significant ( $\mathrm{p}$ value $<0.0001$ ). In-hospital mortality rate (mortality due to any reason during hospitalization for bilateral TKA) was zero in both groups within 2 years of follow-up (Table 1).

Although, overall complication rate (inpatient and/or 90 days readmission) was higher in Group A, but it was statistically not significant. The revision rate of TKAs, in both groups within 2 years of surgery, was zero (Table 2 and Figure 1).
Comparative results of pre-operative and post-operative knee society scores (KSS) in both groups at 1 year are shown (Table 3 and Figure 2).

Radiological and clinical evaluations following the simultaneous bilateral TKA of 2 different cases at different follow-ups are also shown (Figure 3 and 4).

Table 1: Various outcomes of patients in simultaneous and staged bilateral TKA.

\begin{tabular}{|c|c|c|c|}
\hline \multirow[b]{2}{*}{ Various outcomes } & \multicolumn{2}{|l|}{ Bilateral TKA } & \multirow[b]{2}{*}{ P value } \\
\hline & $\begin{array}{l}\text { Simultaneous } \\
\text { (Group A) }\end{array}$ & $\begin{array}{l}\text { Staged } \\
\text { (Group B) }\end{array}$ & \\
\hline Mean surgical time (minutes) & 208.7 & 217.3 & 0.7485 \\
\hline Mean estimated blood loss (ml) & 765.5 & 1090.8 & $<0.0010$ \\
\hline Blood transfusion rate $(\%)$ & 39.4 & 18.1 & $<0.0001$ \\
\hline In-patient length of hospital stays (mean days) & 5.5 & 8.5 & $<0.0001$ \\
\hline In-hospital mortality rate* $(\%)$ & 0.0 & 0.0 & - \\
\hline
\end{tabular}

*In-hospital mortality: mortality due to any reason during hospitalization for bilateral TKA.

Table 2: Overall complication rate (inpatient and/or 90-day readmission) and revision rate of TKAs in both groups within 2 years.

\begin{tabular}{|c|c|c|c|c|}
\hline \multirow{2}{*}{ Various outcomes } & & \multicolumn{2}{|l|}{ Bilateral TKA } & \multirow[b]{2}{*}{ P value } \\
\hline & & $\begin{array}{l}\text { Simultaneous } \\
\text { (Group A) }\end{array}$ & $\begin{array}{l}\text { Staged } \\
\text { (Group B) }\end{array}$ & \\
\hline \multirow{6}{*}{$\begin{array}{l}\text { Overall complication rate } \\
\text { (inpatient and/or 90-day } \\
\text { readmission) (\%) }\end{array}$} & Cardiac complications* & 1.8 & 1.6 & 0.1912 \\
\hline & CNS complications** & 1.5 & 1.3 & 0.3246 \\
\hline & Deep vein thrombosis & 1.6 & 1.2 & 0.1572 \\
\hline & Pulmonary embolism & 1.3 & 1.1 & 0.2689 \\
\hline & Urinary complications $* * *$ & 0.88 & 0.96 & 0.4174 \\
\hline & Knee infection & 0.6 & 1.18 & $<0.0010$ \\
\hline \multirow{2}{*}{ Revision rate of TKAs (\%) } & 1 Year & 0.0 & 0.0 & - \\
\hline & 2 Year & 0.0 & 0.0 & - \\
\hline
\end{tabular}

Cardiac complications*: acute ischemia, infarction, arrhythmia; CNS complications**: cerebrovascular accidents, confusion; Urinary complications $* * *$ : urinary tract infection, anuria.

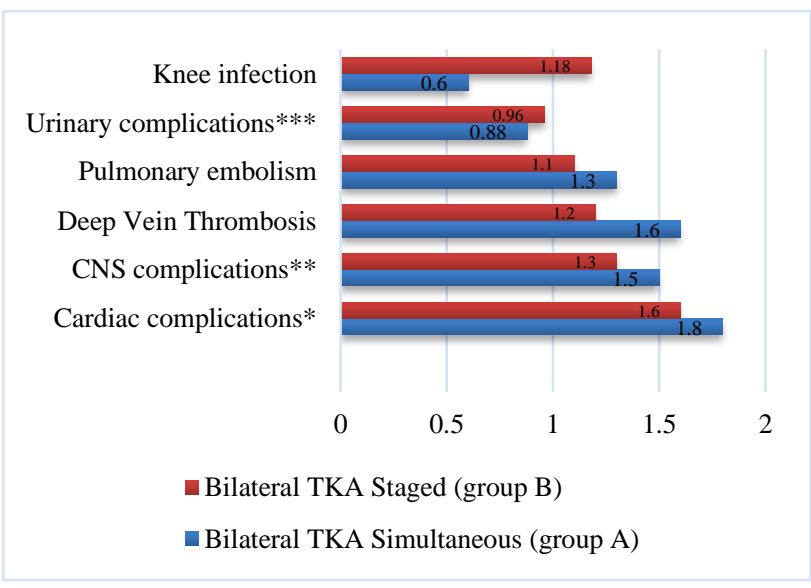

Figure 1: Overall complication rate (inpatient and/or 90 days readmission) in simultaneous and staged bilateral TKA groups.

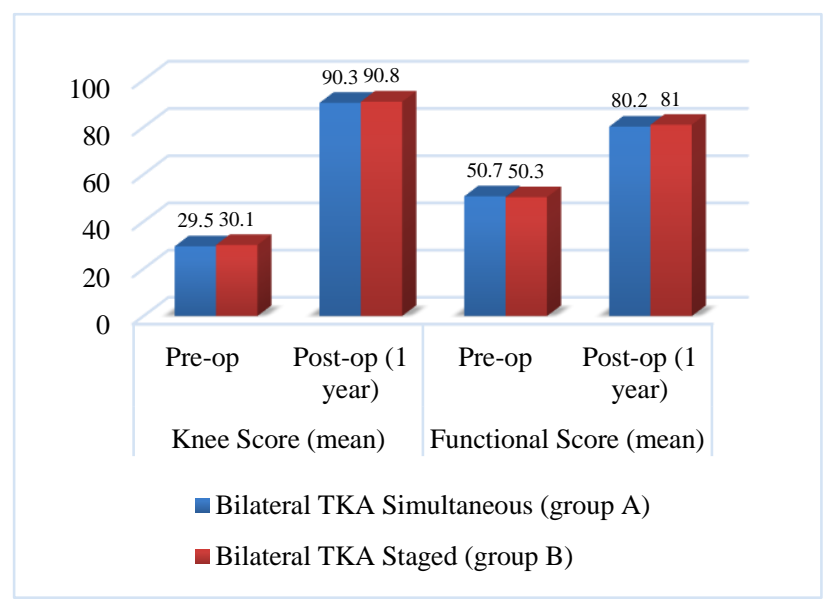

Figure 2: Pre-op and post-op (1 year) knee society score in simultaneous and staged bilateral TKA groups. 


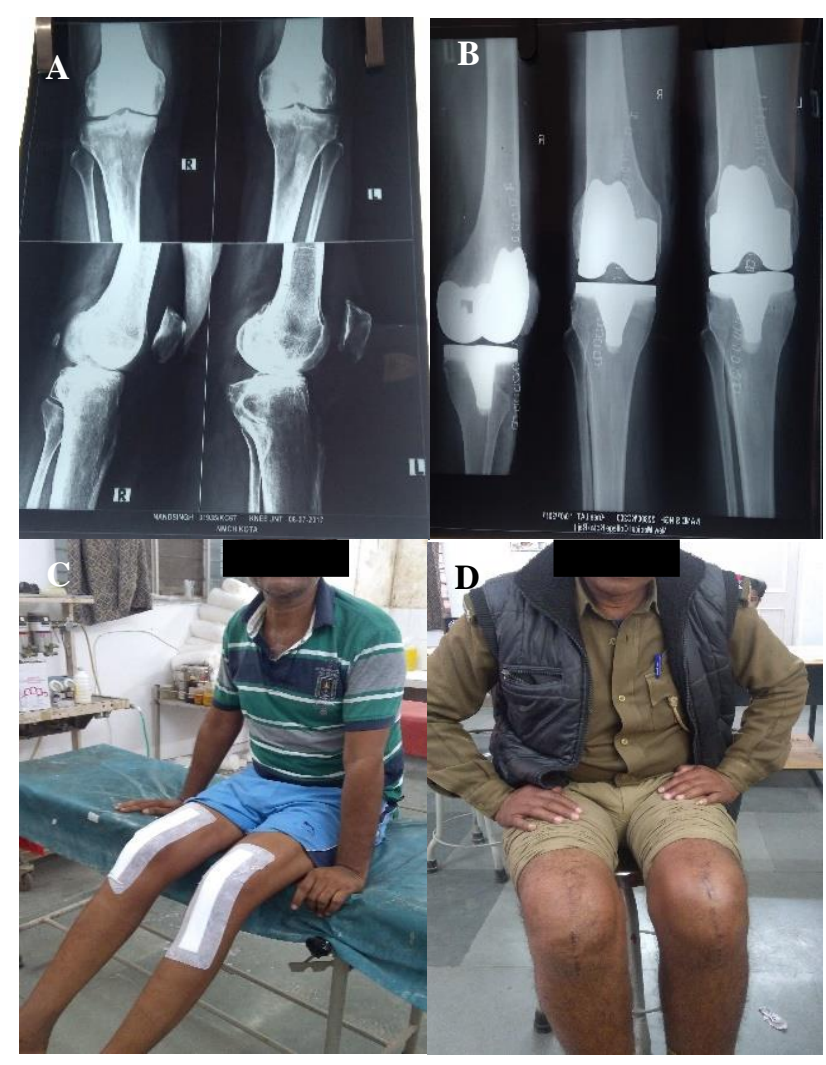

Figure 3: Radiological and clinical evaluation following a simultaneous bilateral TKA. A) preoperative X-ray, B) post-operative X-ray, C) Knee flexion at discharge, D) Surgical scar.

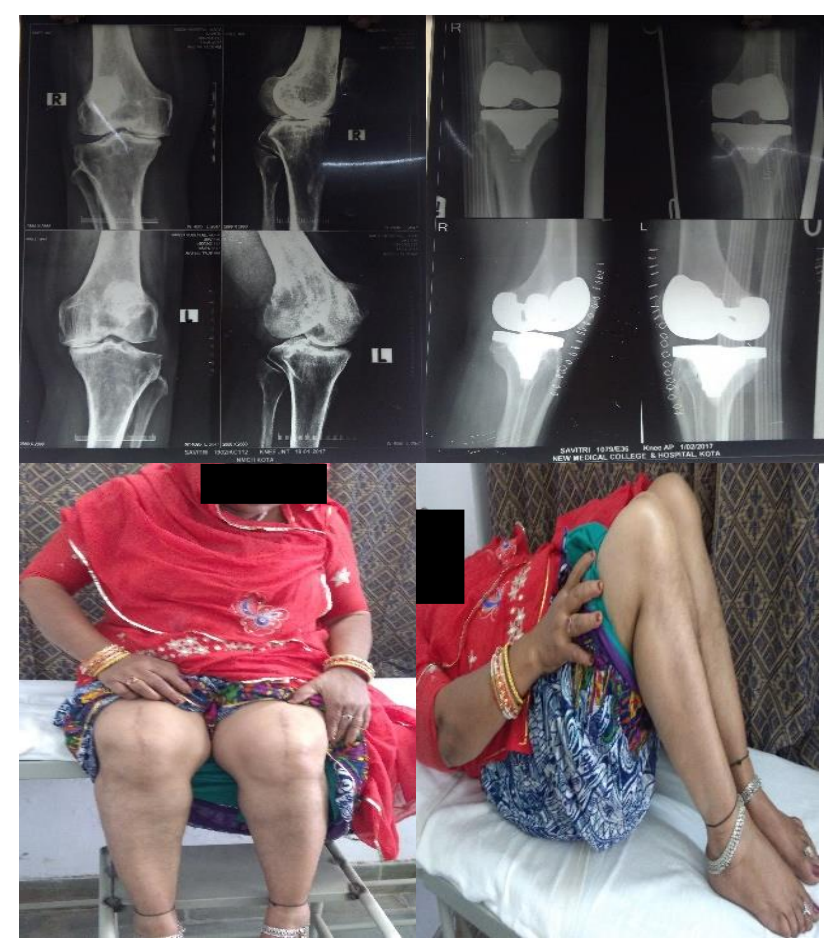

Figure 4: Radiological and clinical evaluation following a simultaneous bilateral TKA. A) preoperative X-ray, B) post-operative X-ray, C) Surgical scar, D) Knee flexion at 6 months post-operative.

\section{DISCUSSION}

TKA is a safe and effective treatment procedure for end stage knee osteoarthritis. It provides significant improvement in the daily routine activities of the patient by improving pain relief, and deformity correction. But in bilateral cases, controversy about the staging of treatment still remains. There are certain risks and benefits of each procedure. According to previous literature, advantages of simultaneous bilateral procedure are single hospital admission, shorter hospital stay, fewer anaesthesia complications, lower combined cost of surgery and fewer days of work loss. ${ }^{6}$ On the other hands, staged bilateral TKA has been associated with less morbidity and mortality; less cardiac and thromboembolic complications. $^{7-9}$

Fewer systemic complications in our study can be explained in this way that most of the cases were ASA grade 1 or grade 2 in our inclusion criteria. These findings are comparable in the literature. ${ }^{10}$ According to Memtsoudis, ASA 3 and 4 patients have a higher risk of severe complications in simultaneous bilateral TKA. ${ }^{11}$

In our study, difference in the surgical time was not statistically significant between the two groups. The mean estimated blood loss was significantly lower in patients with simultaneous procedure (Group A), than in patients who had a staged procedure (Group B), although the transfusion requirements were significantly higher in the group A ( $p$ value $<0.0001$ ). The need for a transfusion was estimated by the post-operative clinical and haematological parameters (haematocrit and hemoglobin levels) which were significantly lower in group A patients. Since the total estimated blood loss in the staged group was the sum of two values during two procedures, carried out with rehabilitation period of three to six months apart, it is easy to understand why the transfusion requirements were lower in these patients. Similar findings have been reported by other studies. ${ }^{7,8}$ Although we did not assess the combined surgical costs, but in the simultaneous group significantly shorter length of hospital stay and early rehabilitation would certainly have an impact in reducing the economic burden. ${ }^{6,7}$

In our study, incidence of DVT and pulmonary embolism were more $(1.6 \%, 1.3 \%$ respectively) in simultaneous bilateral TKA group. These findings are comparable with the literature. ${ }^{12,13}$ Longer time taken in bilateral procedure may increases the risk of DVT by causing stasis of blood. The incidence of perioperative knee infection (1.18\% vs $0.6 \%$ ) was significantly higher in the staged bilateral TKA group ( $p$ value $<0.001$ ). Previous studies accessed that multiple hospitalizations and longer hospital stay were significant predictors for prosthetic joint infection. ${ }^{12,14}$ There was no revision of surgery and no mortality in any of our study group within 2 years of follow-up. However, the number of patients were probably too small to detect any difference between groups. Similar findings by previous studies were reported in the literature. ${ }^{13,15}$ 
One of the limitations of our study was that we compared only low risk group population (ASA grade 1 and 2). We did not take high risk group patients (ASA grade 3 and grade 4) into consideration for evaluation the result. Secondly, the sample size of study population was probably too small to detect any difference between groups.

\section{CONCLUSION}

In conclusion, simultaneous bilateral TKA is as safe as a staged procedure in patients with ASA grade 1 and grade 2. Although the need for transfusion is greater in patients undergoing a simultaneous procedure there is no increase in the incidence of complications. Due to single hospital admission, single anaesthesia session, shorter hospital stays, fewer follow-ups and early rehabilitation, the combined surgical costs are likely to be less in the simultaneous procedure. So, we believe that a simultaneous bilateral TKA is safe, effective and appropriate in our population.

Funding: No funding sources Conflict of interest: None declared

Ethical approval: The study was approved by the local ethics committee

\section{REFERENCES}

1. Gamble R, Ayerst WJ, Johnson EL, Searle WA, Beecham S. Recommendations for the medical management of osteoarthritis of the hip and knee. Arthritis and Rheum. 2000;43(9):1905-15.

2. Brooks PM. Impact of osteoarthritis on individuals and society: how muchdisability. Social consequences and health economic implications. Curr Opin Rheum. 2002;14(5):573-7.

3. Sun BH, Wu CW, Kalunian KC. New developments in osteoarthritis. Rheum Dis Clin N Am. 2007;33(1):135-48.

4. Kim YH, Choi YW, Kim JS. Simultaneous bilateral sequential total knee replacement is as safe as unilateral total knee replacement. J Bone Joint Surg Br. 2009;91:64-8.

5. Carr AJ, Robertsson O, Graves S, Price AJ, Arden NK, Judge A, et al. Knee replacement. Lancet. 2012;379(9823):1331-40.
6. Hutchinson J, Parish E, Cross M. A comparison of bilateral uncemented total knee arthroplasty. Bone Jt J. 2006;88(1):40-3.

7. Lane GJ, Hozack WJ, Shah S, Rothman RH, Booth Jr RE, Eng K, et al. Simultaneous bilateral versus unilateral total knee arthroplasty: outcomes analysis. Clin Orthop Relat Res. 1997;345:106-12.

8. Luscombe J, Theivendran K, Abudu A, Carter S. The relative safety of one-stage bilateral total knee arthroplasty. Int Orthop. 2009;33(1):101-4.

9. Levy YD, Hardwick ME, Copp SN, Rosen AS, Colwell CW. Thrombosis incidence in unilateral vs. simultaneous bilateral total knee arthroplasty with compression device prophylaxis. J Arthroplast. 2013;28(3):474-8.

10. Yoon HS, Han CD, Yang IH. Comparison of simultaneous bilateral and staged bilateral total knee arthroplasty in terms of perioperative complications. J Arthroplasty. 2010;25:179-85.

11. Memtsoudis SG, Ma Y, Chiu YL, Poultsides L, Gonzalez D, Valle A, Mazumdar M. Bilateral total knee arthroplasty: risk factors for major morbidity and mortality. Anesth Analg. 2011;113(4):784-90.

12. Meehan JP, Danielsen B, Tancredi DJ, Kim S, Jamali AA, White RH. A population-based comparison of the incidence of adverse outcomes after simultaneous-bilateral and staged-bilateral total knee arthroplasty. J Bone Jt Surg Am. 2011;93(23):220313.

13. Fu D, Li G, Chen K, Zeng H, Zhang X, Cai Z. Comparison of clinical outcome between simultaneous-bilateral and staged-bilateral total knee arthroplasty: a systematic review of retrospective studies. J Arthroplast. 2013;28(7):1141-7.

14. Poultsides LA, Memtsoudis SG, Vasilakakos T. Infection following simultaneous bilateral total knee arthroplasty. J Arthroplasty. 2013;28:92-5.

15. Sheth DS, Cafri G, Paxton EW. Bilateral simultaneous vs staged total knee arthroplasty: a comparison of complications and mortality. J Arthrop. 2016;31:212-6.

Cite this article as: Sharma RK, Meena RP. Comparison between simultaneous versus staged bilateral total knee arthroplasty: a prospective, randomized, controlled study. Int J Res Orthop 2020;6:562-6. 